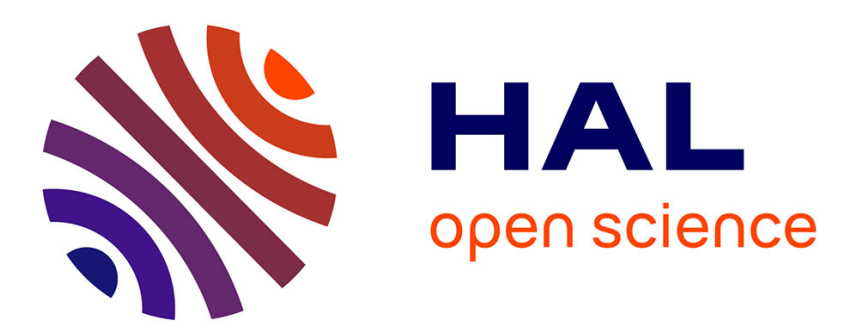

\title{
Mate Limitation in Fungal Plant Parasites Can Lead to Cyclic Epidemics in Perennial Host Populations
}

\author{
Virginie Ravigné, Valérie Lemesle, Alicia A Walter, Ludovic Mailleret,
} Frédéric Marie Hamelin

\section{To cite this version:}

Virginie Ravigné, Valérie Lemesle, Alicia A Walter, Ludovic Mailleret, Frédéric Marie Hamelin. Mate Limitation in Fungal Plant Parasites Can Lead to Cyclic Epidemics in Perennial Host Populations. Bulletin of Mathematical Biology, 2017, 73 (3), pp.1 - 447. 10.1007/s11538-016-0240-7 . hal-01569465

\section{HAL Id: hal-01569465 \\ https://hal.inria.fr/hal-01569465}

Submitted on 26 Jul 2017

HAL is a multi-disciplinary open access archive for the deposit and dissemination of scientific research documents, whether they are published or not. The documents may come from teaching and research institutions in France or abroad, or from public or private research centers.
L'archive ouverte pluridisciplinaire $\mathbf{H A L}$, est destinée au dépôt et à la diffusion de documents scientifiques de niveau recherche, publiés ou non, émanant des établissements d'enseignement et de recherche français ou étrangers, des laboratoires publics ou privés.

\section{다(1)(2)}

Distributed under a Creative Commons Attribution - ShareAlike| 4.0 International 


\title{
Mate limitation in fungal plant parasites can lead to cyclic epidemics in perennial host populations
}

\author{
Virginie Ravigné · Valérie Lemesle · \\ Alicia Walter · Ludovic Mailleret . \\ Frédéric M. Hamelin
}

Received: date / Accepted: date

\begin{abstract}
Fungal plant parasites represent a growing concern for biodiversity and food security. Most ascomycete species are capable of producing different types of infectious spores both asexually and sexually. Yet the contributions of both types of spores to epidemiological dynamics have still to been fully researched. Here we studied the effect of mate limitation in parasites which perform both sexual and asexual reproduction in the same host. Since mate limitation implies positive density dependence at low population density, we modeled the dynamics of such species with both density-dependent (sexual) and density-independent (asexual) transmission rates. A first simple SIR model incorporating these two types of transmission from the infected compartment, suggested that combining sexual and asexual spore production can generate persistently cyclic epidemics in a significant part of the parameter space. It was then confirmed that cyclic persistence could occur in realistic situations by parameterizing a more detailed model fitting the biology of the Black Sigatoka disease of banana, for which literature data is available. We discuss the implications of these results for research on and management of Sigatoka diseases of banana.
\end{abstract}

Keywords mixed mating system, fungi, epidemiology, nonlinear transmission, limit cycle, competitive exclusion, Allee effect, sexual reproduction, asexual reproduction

Mathematics Subject Classification (2000) 92D25 · 92D30 · 34A99

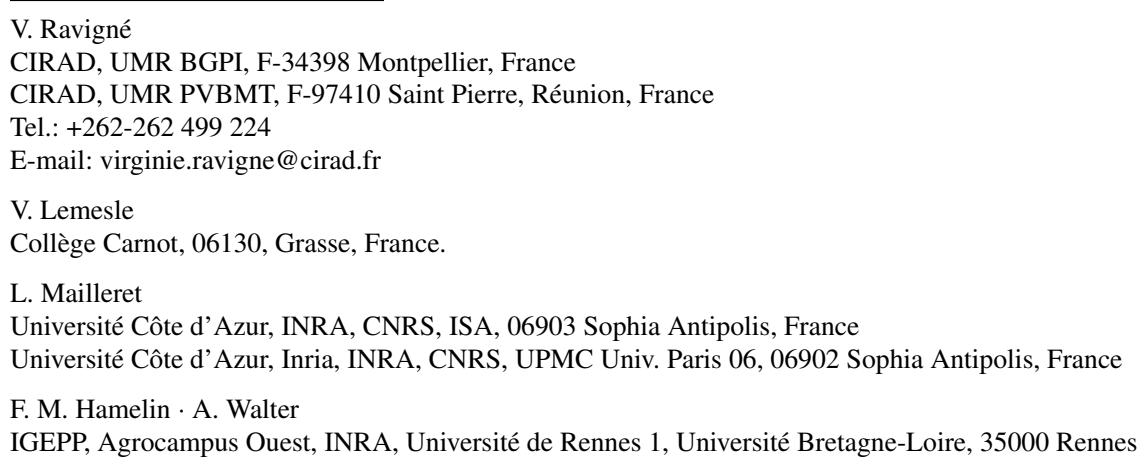




\section{Introduction}

Fungal plant parasites represent a growing concern for biodiversity and food security (Pennisi 2010, Fisher et al. 2012). In many fungi (mainly ascomycete species but also in other groups) the asexual production of infectious spores is complemented by the possibility to produce spores through the fusion of haploid individuals and meiosis, i.e., sexual reproduction. Epidemics are thus propagated by two different types of spores often produced from the same hosts. Sexually produced spores often have higher propagative abilities than asexually produced spores, be it in terms of dispersal, survival, or both (Bonner, 1958; Williams, 1975). Moreover, in most of these fungi, the successful fusion of gametes can only occur between haploids carrying functionally different mating-type alleles (hereafter + and - ), a phenomenon called heterothallism (Billiard et al, 2011). The production of spores through sexual reproduction therefore requires that sufficient density is attained for two sexually compatible pathogens to meet. It is thus exposed to mate limitation at low density.

Numerous plant pathogens, some of which are considered serious threats to global food security, are heterothallic. The South American leaf blight of rubber tree, historically known as having ruined Ford's industrial production of latex in Brazil by devastating local Hevea plantations in the 40s, is caused by the ascomycete Microcyclus ulei, presumably heterothallic (Watling et al, 2002). The Gibberella fujikuroi species complex counts at least eight different heterothallic species pathogenic on pine, maize, rice, banana in both temperate and tropical conditions, with massive socio-economic effects (Steenkamp et al, 2000; Nirenberg and O'Donnell, 1998). Colletotrichum lindemuthianum, responsible for black spot disease of common bean, is considered heterothallic as well as many strains in the Colletotrichum genus (RodríguezGuerra et al, 2005; Cannon et al, 2012). More generally, with the advent of genomes studies, more and more species once classified as asexual, are now recognized as having retained the machinery for sexual reproduction and in some cases as having operational heterothallism genes (Ene and Bennett, 2014).

Despite the importance of these species for human welfare, there are very few studies on the effect of sexual reproduction on epidemiological dynamics (Castel et al, 2014). Epidemiological dynamics have traditionally been modeled assuming clonal reproduction of the parasite. Models accounting for sexual reproduction in parasites mostly focused on helminth-like life histories, with obligate sexual reproduction in one of several hosts. Importantly, these models showed that mate limitation may give rise to demographic Allee effects and epidemiological thresholds (May, 1977; Anderson and May, 1981; Nåsell et al, 1985; May and Woolhouse, 1993; Cornell et al, 2004). Nevertheless, the specifics of helminth life cycles make these models inadequate for most fungi. There is currently no generic epidemiological model for species able to produce distinct asexual and sexual spores from the same host, hampering the understanding and control of these pathogens.

A good example of diseases where improved understanding of the epidemiological roles of asexually and sexually produced spores would be helpful is given by Mycosphaerella musicola and Mycosphaerella fijiensis, respectively responsible for the Yellow and Black Sigatoka banana diseases. The Yellow Sigatoka, caused by $M$. musicola was first identified in Java in 1902. It rapidly spread throughout the whole 
intertropical belt. The Black Sigatoka disease, caused by M. fijiensis, appeared in red the Fiji islands in 1963. In the 70s, it was reported in Central America (Honduras) and in Africa (Gabon). Since 2011, it has been present in most tropical banana-producing areas, where it seems to have replaced M. musicola. Both diseases cause huge economic losses (i) through important defoliations and subsequent yield loss, (ii) by compromising banana export through their precocious overripening, and (iii) because only fungicide applications are considered efficient in controlling the disease. Both species infect the main commercial banana varieties and share the same life cycle (Figure 3). Spores deposit at the surface of young banana leaves at the top of the canopy where they germinate and form mycelia. When a mycelium penetrates the leaf epiderm, through stomata, it forms a lesion and grows by consuming plant cells around. After a latency stage, the lesion turns mature and produces structures (conidiophores) bearing asexual spores (conidia). These spores are dispersed by rain-splash and infect plant tissues as much as a few meters (Rieux et al, 2014). As lesions keep on growing they form large necrotic areas and turn sexually mature. At this stage, if two mycelia of compatible mating types are large and close enough on the same leaf, they engage in sexual reproduction by producing specific structures (perithecia and spermogonia) and spores (ascospores). Sexual spores are then dispersed by wind from several hundred meters to several kilometers (Rieux et al, 2014). In all types of established plantations, banana trees regularly produce new leaves in a non synchronous manner providing the parasite with an fairly constant input of healthy tissues to infect. $M$. fijiensis produces fewer asexual spores and more sexual spores than M. musicola, so that it has sometimes been suggested that the ecological superiority of $M$. fijiensis was rooted in its greater investment in sexual reproduction (Stover, 1980; Fouré, 1982). However, due to a lack of adequate epidemiological models, we have only limited understanding of the contributions of both types of spores to the epidemiological dynamics of both diseases.

Aims and outline of the study

The first objective of the present study was to explore how epidemiological dynamics are affected by the ability to produce both asexual and sexual spores from a single infected host. To do so, we built and analyzed a simple and generic SusceptibleInfected-Removed (SIR) model incorporating the key specifics of heterothallic fungal parasites, i.e., that disease transmission relies on two distinct types of infectious spores respectively produced asexually and sexually from the infected compartment. The effects of this nonlinear density-dependence on epidemic thresholds, and longterm epidemiological dynamics were documented using analytical and numerical bifurcation analyses. It was especially shown that sexually-produced spores could cause sustained oscillations of disease prevalence. The second objective of the paper was thus to evaluate whether the parameter range leading to such oscillating epidemics was likely to be encountered in nature. To implement this, a more detailed model was built, which accounts for the precise sequencing of stages that lesions go through after Sigatoka infection occurred. The detailed model was parameterized using literature data for the Sigatoka banana diseases (Robert et al, 2012), and its epidemiological dy- 
namics were compared to the simple model. We discussed the theoretical importance of our findings and highlight some perspectives for research on Sigatoka diseases.

\section{Simple model}

Here we study a simple SIR model incorporating the key specifics of heterothallic fungal parasites i.e. that disease transmission relies on two distinct types of infectious spores respectively produced asexually and sexually from the infected compartment.

We looked at an environment in which host plants are continuously present. The term 'site' hereafter designates an infection site, i.e., a part of leaf tissue where an infection may take place and where a lesion may develop (Zadoks, 1971; Gourbière and Gourbière, 2002; Savary et al, 2015), so that multiple infections cannot occur in the model. Let $S(t), I(t)$ and $R(t)$ be respectively the densities of susceptible, infected and removed (no longer infectious) sites at time $t$. The removal rate of infected sites is $\alpha$. Total site density is $N=S+I+R$. Let $\phi$ be the mean one-sided site area, so that $\phi N$ is the so-called Leaf Area Index (LAI) (Jordan, 1969). The spatial arrangement of hosts and spore dispersal distances are left implicit in this study, the latter point being addressed in a companion paper (Hamelin et al, 2016).

\subsection{Host population dynamics}

Host dynamics, i.e., the rate of emission of new leaf tissues, are assumed disease independent. Let $\mu$ be basic leaf mortality rate and $f(N)$ be their renewal rate, which includes natural host dynamics and possible agricultural practices. We introduce the notation $\dot{N}$ to denote the derivative of $N$ with respect to time $t$. Host dynamics follow $\dot{N}=f(N)-\mu N$. Let $\bar{N}$ be equilibrium host density, i.e. $\bar{N}$ is such that $f(\bar{N})=\mu \bar{N}$. We assume that host renewal is such that the host has stable population dynamics, i.e. $f^{\prime}(\bar{N})<\mu$. From now on, $N$ is assumed to be at equilibrium $(N=\bar{N})$.

\subsection{Asexual dynamics}

Let $b$ denote the number of asexual spores (conidia) produced per infected site per unit time, and $0<r<1$ be their infectivity, that is, the probability that an asexual spore in contact with a susceptible site succeeds in infecting it.

We are interested in deriving the average number of spores deposited on an individual site, within a short time, given spore density. A fully detailed model would take into account the architecture of vegetation cover. For the sake of producing an analytically tractable and clear formulation, we consider two possible simplifications. Firstly, let us suppose that leaves do not overlap. The canopy is open. Total leaf area is smaller than ground area and therefore LAI is lower than 1: $\phi N \leq 1$. Secondly, that leaves overlap up to the point that canopy is closed. Then $\phi N>1$. In brief, per capita exposed site area is either $\phi$ (if $\phi N \leq 1$ ) or $1 / N$ (if $\phi N>1$ ). That is to say, if the canopy is open and leaves do not overlap, then each site exposes its entire one-sided area to spore landing. If the canopy is closed and leaves overlap 
homogeneously, then each site exposes an equal part $1 / N$ of the total area to spore landing. Thus, within a time interval $(t, t+\mathrm{d} t)$, small enough to neglect new infections, the mean number of spores received per site is either (i) $\phi b I(t) \mathrm{d} t$ (if $\phi N \leq 1$ ) or (ii) $(1 / N) b I(t) \mathrm{d} t$ (if $\phi N>1$ ). Hence, the mean number of spores received per site is $\min (\phi, 1 / N) b I(t) \mathrm{d} t$. Let $X$ be a random variable that accounts for the number of spores received by a site during this short time, i.e. $X$ is an integer whose mean is $\mathbb{E} X=\min (\phi, 1 / N) b I(t) \mathrm{d} t \ll 1$.

Let

$$
\beta=\frac{\operatorname{br} \min (\phi N, 1)}{N}
$$

The probability for a susceptible site to escape infection is $\mathbb{E}(1-r)^{X}$. Assuming that $r \ll 1$ (low infectivity), a Taylor approximation of $(1-r)^{X}$ in $r=0$ yields $1-r X$, so that the probability of escaping infection during $\mathrm{d} t$ is approximately $1-r \mathbb{E} X=1-$ $\beta I(t) \mathrm{d} t$. We thus get $S(t+\mathrm{d} t)=[1-\beta I(t) \mathrm{d} t] S(t)$. Assuming that $\mathrm{d} t$ is infinitesimal, asexual disease dynamics reads

$$
\dot{S}=-\beta S I
$$

which corresponds to a classical SI model.

Note that the above assumptions yield (i) $\dot{S}=-\phi b r \times S I$ or (ii) $\dot{S}=-b r \times S I / N$, i.e., density- and frequency-dependent transmission rates, also called mass-action and proportional incidences, respectively (McCallum et al, 2001; Arino and McCluskey, 2010). Transmission is frequency-dependent in closed canopies $(\phi N>1)$ and density-dependent in open canopies $(\phi N<1)$.

\subsection{Sexual dynamics}

In many heterothallic diseases, sexual reproduction occurs through a direct contact between two adjacent lesions of compatible mating types. To simplify, we assume an even sex-ratio. Let $s$ be the number of sexual spores (ascospores) produced per infected site per unit time, provided that two infected sites with compatible mating types meet. Let $0<q<1$ be the infectivity of sexual spores. Let $n$ be the mean number of neighboring sites surrounding any given site. The mean number of infected sites in the neighboring area of any given site is $n(I / N)$.

Assuming that the spatial distribution of infected sites is random, the number of compatible partners in the contact area of a given site, i.e. the neighboring sites, follows a Poisson distribution with parameter $n I /(2 N)$, the division by 2 accounting for a balanced sex ratio. Therefore, the probability of having at least one compatible partner in the contact area is

$\mathbb{P}$ (number of compatible partners in the contact area $\geq 1)$

$=1-\mathbb{P}($ number of compatible partners in the contact area $=0)$,

$=1-\exp (-n I /(2 N))$,

$\approx n \frac{1}{2} \frac{I}{N}$ 
provided that the argument of the exponential is small. This is a reasonable assumption since $I /(2 N) \ll 1$.

Proceeding as in Section 2.2, but conditioning sexual spores production when there is a compatible mating partner, we end up with

$$
\dot{S}=-\underbrace{n \frac{1}{2} \frac{I}{N}}_{\text {mating }} \times \underbrace{s q I}_{\begin{array}{c}
\text { infectious sexual } \\
\text { spores emission }
\end{array}} \times \underbrace{\min (\phi, 1 / N)}_{\begin{array}{c}
\text { area exposed to spore } \\
\text { landing (per site) }
\end{array}} \times S=-\frac{n s q \min (\phi N, 1)}{2 N^{2}} \times S I^{2},
$$

which corresponds to a SI model with non-linear transmission terms (Liu et al, 1986). Let

$$
\sigma=\frac{n s q \min (\phi N, 1)}{2 N^{2}}
$$

\subsection{Mixed sexual-asexual dynamics}

Lesions are identical regardless as to whether they were created by sexual or asexual spores. When we merge, Sections 2.1, 2.2, and 2.3, and assume that only susceptible hosts are introduced into the system, the simple model reads:

$$
\begin{aligned}
\dot{S} & =\mu N-\beta S I-\sigma S I^{2}-\mu S, \\
\dot{I} & =\beta S I+\sigma S I^{2}-(\alpha+\mu) I, \\
\dot{R} & =(\alpha+\mu) I-\mu R .
\end{aligned}
$$

For all $t, R(t)=N-S(t)+I(t)$, so that we hereafter leave the $R$ equation implicit.

\subsection{Non-dimensionalization}

Let

$$
S^{*}=\frac{S}{N}, \quad I^{*}=\frac{I}{N}, \quad t^{*}=(\alpha+\mu) t
$$

and

$$
\mu^{*}=\frac{\mu}{\alpha+\mu}, \quad \beta^{*}=\frac{\beta N}{\alpha+\mu}, \quad \sigma^{*}=\frac{\sigma N^{2}}{\alpha+\mu} .
$$

Model (4) becomes

$$
\begin{aligned}
& \frac{\mathrm{d} S^{*}}{\mathrm{~d} t^{*}}=\mu^{*}-\beta^{*} S^{*} I^{*}-\sigma^{*} S^{*} I^{* 2}-\mu^{*} S, \\
& \frac{\mathrm{d} I^{*}}{\mathrm{~d} t^{*}}=\beta^{*} S^{*} I^{*}+\sigma^{*} S^{*} I^{* 2}-I^{*},
\end{aligned}
$$

so that the model behavior is entirely determined by the 3 parameters $\mu^{*}, \beta^{*}$, and $\sigma^{*}$. 
2.6 Epidemic threshold

Let $G$ be the Jacobian matrix associated with (8):

$$
G=\left(\begin{array}{cc}
-\beta^{*} I^{*}-\sigma^{*} I^{* 2}-\mu^{*} & -\beta^{*} S^{*}-2 \sigma^{*} S^{*} I^{*} \\
\beta^{*} I^{*}+\sigma^{*} I^{* 2} & \beta^{*} S^{*}+2 \sigma^{*} S^{*} I^{*}-1
\end{array}\right) .
$$

Linearizing around the Disease Free Equilirium (DFE) $\left(S^{*}, I^{*}\right)=(1,0)$, we get

$$
G_{(0,0)}=\left(\begin{array}{cc}
-\mu^{*} & -\beta^{*} \\
0 & \beta^{*}-1
\end{array}\right)
$$

whose eigenwalues are $\Lambda_{1}=-\mu^{*}<0$ and $\Lambda_{2}=\beta^{*}-1$. Hence the DFE is asymptotically stable if and only if

$$
\mathscr{R}_{0}=\beta^{*}<1
$$

Note that $\mathscr{R}_{0}$ does not depend on sexual transmission rate $\sigma$ and is the same as expected under SIR dynamics without sexual reproduction (Anderson and May, 1981). When $\mathscr{R}_{0}>1$, the parasite population initially grows. Conversely, when $\mathscr{R}_{0}<1$, an initially infinitesimal pathogen population cannot grow. Nevertheless, an initially rare, but not infinitesimal, population may grow even for $\mathscr{R}_{0}<1$ thanks to the positive density dependence in the parasite growth rate induced by sexual reproduction, as already observed for instance by Nåsell (1978) and Reluga et al (2008).

\subsection{Long-term dynamics}

The long-term dynamics of diseased leaves depend greatly on the balance between sexual and asexual reproduction. After initial growth, a strictly asexual parasite $\left(\sigma^{*}=\right.$ 0 ) reaches a stable endemic equilibrium provided $\mathscr{R}_{0}>1$ (Anderson and May, 1981). The strictly sexual case $\left(\beta^{*}=\mathscr{R}_{0}=0\right)$ is a particular instance of a model by Liu et al (1986), who considered nonlinear incidence rates: in this case, cyclic persistence is possible.

To explore the possible dynamics brought about by mixed reproduction systems as described in equation (8), a bifurcation analysis was conducted using MatCont v5.2 (Dhooge et al, 2008) on Matlab R2013a (The Mathworks Inc. 2013). We explore the parameter space where $\mu^{*}>0$ and $\sigma^{*}>0$ for different values of $\beta^{*}$ representing three different generic cases: (i) strictly sexual reproduction $\left(\beta^{*}=0\right)$, (ii) low asexual reproduction $\left(\beta^{*}=\mathscr{R}_{0}=0.8<1\right)$, (iii) high asexual reproduction $\left(\beta^{*}=\mathscr{R}_{0}=2>\right.$ 1). Local and global co-dimension 1 and 2 bifurcations are researched (Kuznetsov, 1998). The analysis shows that, for strictly sexual species, cyclic persistence is not a generic outcome (Figure $2 \mathrm{~A})$. Under mixed reproduction $\left(\sigma^{*}>0, \beta^{*}>0\right)$, the range of parameters for which cyclic persistence is possible increases (Figure 2B) and becomes significant when $\mathscr{R}_{0}>1$ (Figure $2 \mathrm{C}$ and 1 ). 


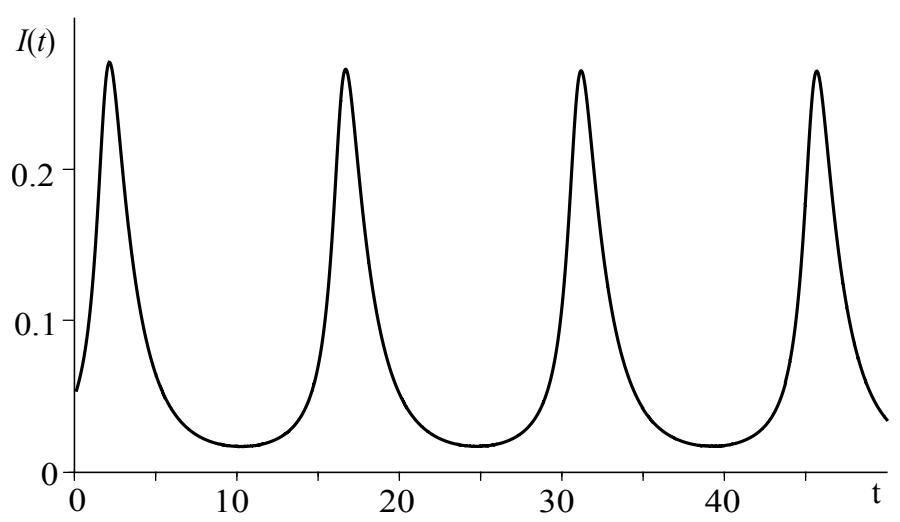

Fig. 1 Mixed mating may lead to cyclic persistence of parasites. Dimensionless model (8) with parameters $\sigma^{*}=25, \mu^{*}=0.1$, and $\beta^{*}=2$.

\section{Sigatoka diseases of bananas}

In what follows, we adapt the simple model to the biology of the Sigatoka diseases of bananas and explore whether sustained oscillations may occur in realistic parameter ranges.

The life cycle of the causal agents of the Sigatoka diseases, M. musicola and $M$. fijiensis, is depicted in Figure 3. For these and many other fungal plant diseases, leaf susceptibility decreases over time so that most infections occur by spore deposition and germination on young leaves. After germination, lesions grow and go through a series of stages: latent, producing asexual spores, latent again, and producing sexual spores (Stover, 1980; Robert et al, 2012). The epidemiological literature provides durations of these stages, spore production rates and spore infectivities.

The full model is represented in Figure 4. The infected compartment, in density $I(t)$, was decomposed into four sub-compartments, so that $I=E_{a}+I_{a}+E_{s}+I_{s}$. Infections initially produce latent lesions, in density $E_{a}$, unable to produce spores for a mean time period of $\gamma_{a}^{-1}$ time units (asexual latency). The next stage encompasses lesions, in density $I_{a}$, producing asexual spores. This stage lasts $\alpha_{a}^{-1}$ time units on average (asexual infectious period). The next stage, in density $E_{S}$, encompasses lesions that have finished producing asexual spores but are not yet sexually mature. This stage lasts $\gamma_{s}^{-1}$ (sexual latency). After this stage lesions, in density $I_{s}$, produce sexual spores during $\alpha_{s}^{-1}$ time units (sexual infectious period).

The full model reads:

$$
\begin{aligned}
\dot{S} & =\mu N-\beta S I_{a}-\sigma S I_{s}^{2}-\mu S \\
\dot{E}_{a} & =\beta S I_{a}+\sigma S I_{s}^{2}-\left(\gamma_{a}+\mu\right) E_{a} \\
\dot{I}_{a} & =\gamma_{a} E_{a}-\left(\alpha_{a}+\mu\right) I_{a} \\
\dot{E}_{s} & =\alpha_{a} I_{a}-\left(\gamma_{s}+\mu\right) E_{s} \\
\dot{I}_{s} & =\gamma_{s} E_{s}-\left(\alpha_{s}+\mu\right) I_{s} . \\
\dot{R} & =\alpha_{s} I_{s}-\mu R .
\end{aligned}
$$




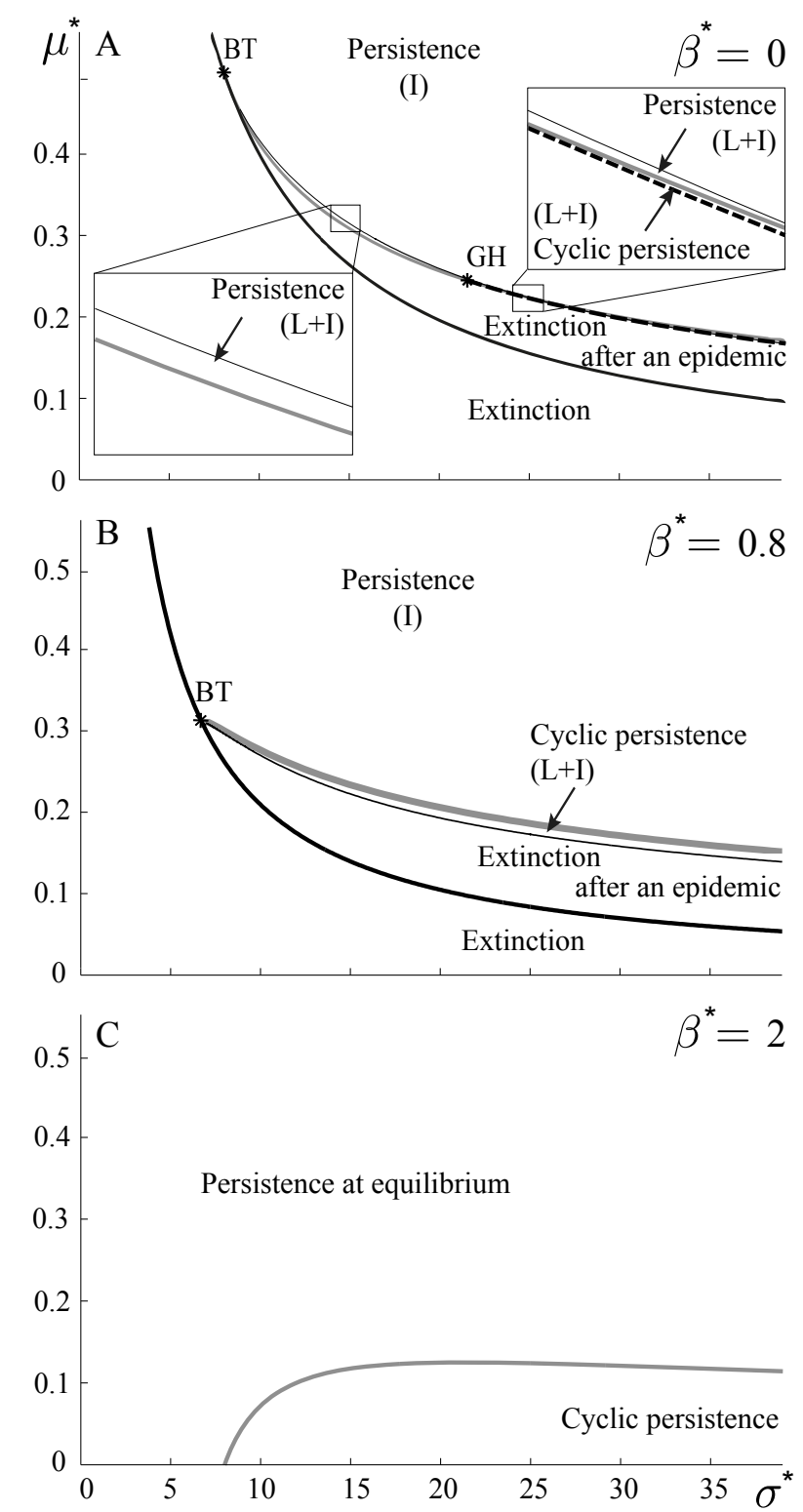

Fig. 2 Bifurcation analysis of the dimensionless model (8) as a function of sexual transmission $\left(\sigma^{*}\right)$ and host basic renewal rate $\left(\mu^{*}\right)$ for three pathogen types: strictly sexual $\left(\beta^{*}=0\right.$, panel A), mixed reproduction with low basic reproduction number $\left(\mathscr{R}_{0}=\beta^{*}=0.8\right.$, panel $\left.\mathrm{B}\right)$, and mixed reproduction with high basic reproduction number $\left(\mathscr{R}_{0}=\beta^{*}=2\right.$, panel $\left.\mathrm{C}\right)$. The stars indicate co-dimension 2 bifurcations (BT: Bogdanov Takens; GH: Generalized Hopf). These points give rise to curves locating co-dimension 1 bifurcations (black thick line: fold; gray thick line: Poincaré-Andronov-Hopf), and global bifurcations (black thin line: homo-clinic bifurcation; black dashed line: fold bifurcation of periodic orbits). Parasite extinction is direct below the black thick curve, and may be preceded by a single epidemic between the black thick curve and the other curves. Above any curve, the parasite can persist at equilibrium although its low asexual capacities combined with low initial densities still drives it to extinction (panels A, B). Cyclic persistence is possible but not generic for strictly sexual species (panel A); the conditions for cyclic persistence get less and less stringent as asexual capacities increase (panel B, C). To best describe the possible dynamics in the figure, we used plain text with the following code: I means that persistence is conditional on initial densities, and L means that the set of initial conditions leading to persistence is restricted nearby the persistence equilibrium or cycle; otherwise the parasite goes extinct. 


\section{Healthy leaves}

\section{Diseased leaves}

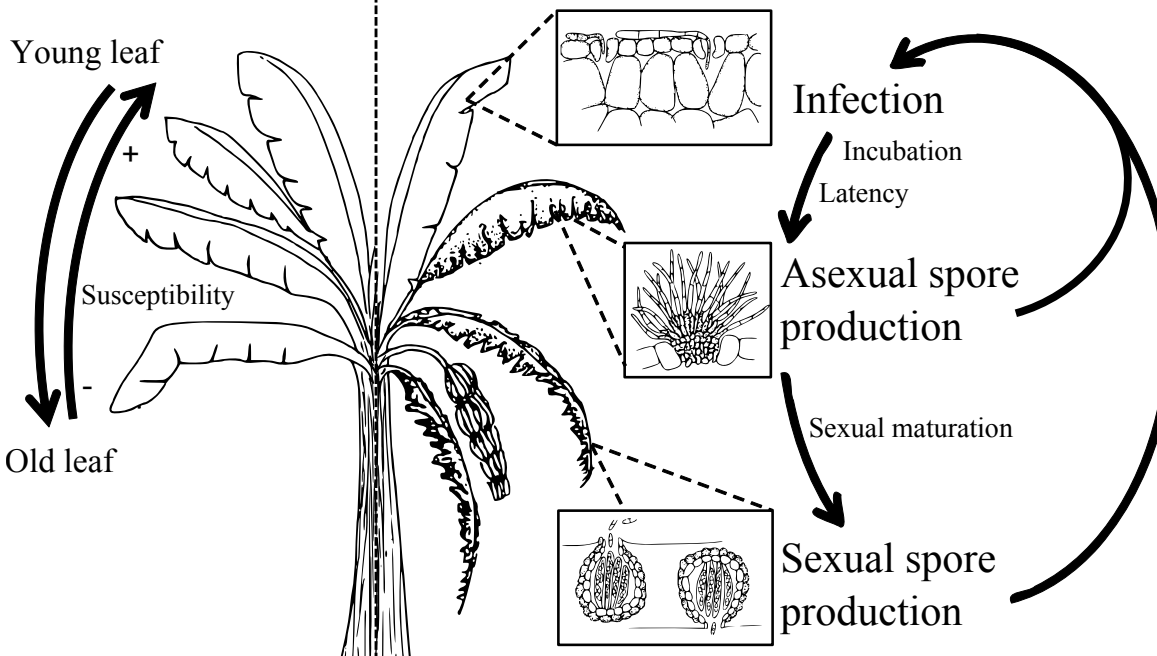

\section{Pathogen life cycle}

Fig. 3 The development of Sigatoka diseases on a banana tree. Left. Approximately once a week, a young banana leave deploys at the top of the trunk and develops for several weeks, going down as new leaves are produced. Susceptibility to infection is maximal upon leaf emergence and decreases over time. Right. Infection occurs as spores deposit on leaves and germinate into stomata. Lesions develop by mycelial growth. After a latent stage, they start producing asexual spores, called conidia, in specific structures called conidiophores (M. fijiensis) or sporodochia (M. musicola). Meanwhile lesions keep on growing to form large necrotic areas. At some point these become sexually mature and sexual reproduction may occur between two adjacent mycelia, in structures called pseudothecia, resulting in ascospores. Both conidia and ascospores can initiate new lesions. Modified from Agrios (2005).

\subsection{Epidemic threshold}

Let $J$ be the Jacobian matrix associated with (10):

$$
J=\left(\begin{array}{ccccc}
-\beta I_{a}-\sigma I_{s}^{2}-\mu & 0 & -\beta S & 0 & -2 \sigma S I_{s} \\
\beta I_{a}+\sigma I_{s}^{2} & -\left(\gamma_{a}+\mu\right) & \beta S & 0 & 2 \sigma S I_{s} \\
0 & \gamma_{a} & -\left(\alpha_{a}+m u\right) & 0 & 0 \\
0 & 0 & \alpha_{a} & -\left(\gamma_{s}+\mu\right) & 0 \\
0 & 0 & 0 & \gamma_{s} & -\left(\alpha_{s}+\mu\right)
\end{array}\right) .
$$

Linearizing around the DFE $(N, 0,0,0,0)$, we get

$$
J_{(N, 0,0,0,0)}=\left(\begin{array}{ccccc}
-\mu & 0 & -\beta N & 0 & 0 \\
0 & -\left(\gamma_{a}+\mu\right) & \beta N & 0 & 0 \\
0 & \gamma_{a} & -\left(\alpha_{a}+m u\right) & 0 & 0 \\
0 & 0 & \alpha_{a} & -\left(\gamma_{s}+\mu\right) & 0 \\
0 & 0 & 0 & \gamma_{s} & -\left(\alpha_{s}+\mu\right)
\end{array}\right)
$$



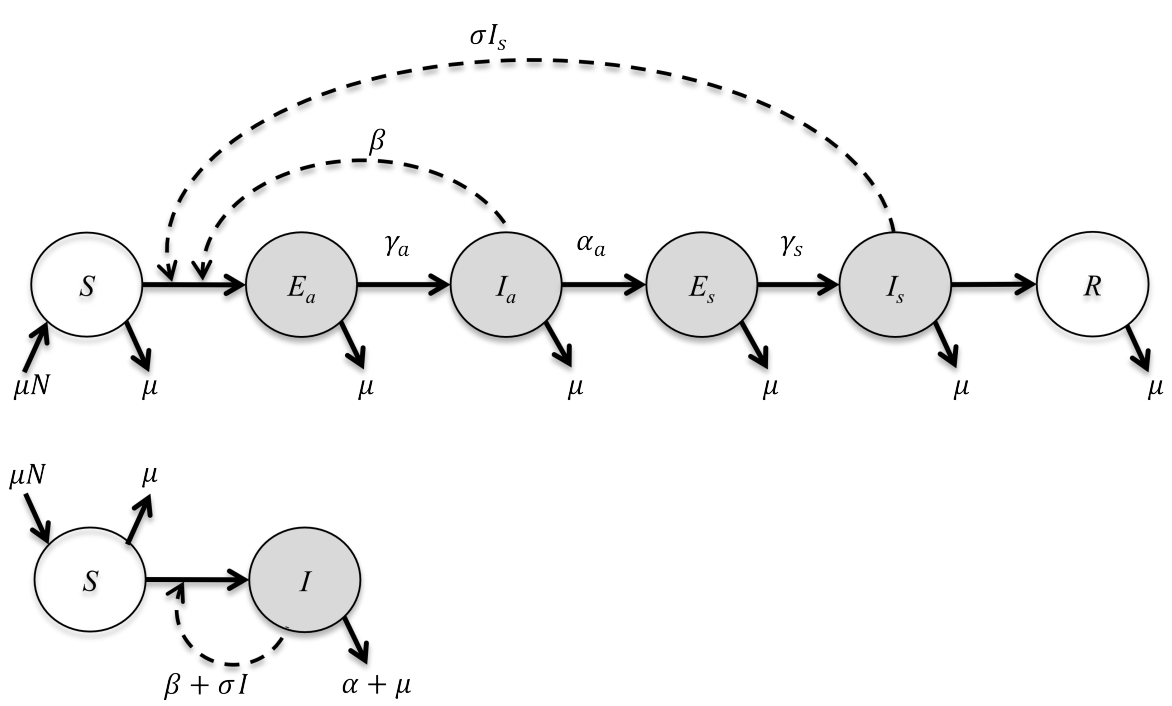

Fig. 4 Epidemiological models for diseases with both sexually and asexually produced infectious spores Top. The full model inspired by the life cycle of Sigatoka diseases. Bottom. The generic SIR model. Parameter notations are defined in the text.

whose eigenwalues are $\lambda_{1}=-\mu<0, \lambda_{2}=-\left(\gamma_{s}+\mu\right)<0, \lambda_{3}=-\left(\alpha_{s}+\mu\right)<0$,

$$
\begin{aligned}
& \lambda_{4}=-\frac{1}{2}\left(\alpha_{a}+\gamma_{a}+2 \mu+\sqrt{4 \beta N \gamma_{a}+\left(\alpha_{a}-\gamma_{a}\right)^{2}}\right)<0, \\
& \lambda_{5}=-\frac{1}{2}\left(\alpha_{a}+\gamma_{a}+2 \mu-\sqrt{4 \beta N \gamma_{a}+\left(\alpha_{a}-\gamma_{a}\right)^{2}}\right) .
\end{aligned}
$$

Hence the DFE is asymptotically stable if and only if $\lambda_{5}<0$, which can equivalently be expressed as

$$
\mathscr{R}_{0}=\frac{\gamma_{a}}{\gamma_{a}+\mu} \frac{\beta N}{\alpha_{a}+\mu}<1
$$

Again, the basic reproductive number $\mathscr{R}_{0}$ is the same as in a simple SEIR model, see e.g. (van den Driessche and Watmough, 2008). In particular, it does not depend on the parameters related to sexual reproduction, $\alpha_{s}, \gamma_{s}$, and $\sigma$.

\subsection{Parameterization for Black Sigatoka}

We now parameterize the model using literature data concerning the Black Sigatoka disease of banana. We only focus on orders of magnitude here, as full parameterization would require further experiments.

In a commercial banana plantation, the Leaf Area Index (Jordan, 1969) ranges from 2 to 5 (Turner et al, 2007). In that case, with LAI $\phi N$ greater than one, equations (1) and (3) yield

$$
\beta=\frac{b r}{N}, \quad \text { and } \quad \sigma=\frac{n s q}{2 N^{2}}
$$


As the model is spatially homogeneous, we chose a space unit so that $N=1$ without loss of generality.

Banana trees deploy a new leaf each week. A leaf stays green on the banana trees for about 12 weeks. Then it dries and falls on the ground, where, depending on agricultural practices, it may or may not be removed. Asexual latency lasts for about 20 days (Stover, 1980; Fouré, 1982) so we let $\gamma_{a}=0.05$ per day. Asexual infectious period is about 10 days (Robert, 2012; Landry, 2015) so we let $\alpha_{a}=0.1$ per day. Sexual latency period is estimated to be around 10 days (Stover, 1980) so we let $\gamma_{s}=0.1$ per day. Sexual infectious period is around 25 days (Stover, 1980) so we let $\alpha_{s}=0.04$ per day. Infection efficiency is about $1 \%$ for both sexual and asexual spores (Landry, 2015), so we let $q=r=0.01$. M. fijiensis produces about 10 conidia (asexual spores) per $\mathrm{mm}^{2}$, and 200 ascospores (sexual spores) per $\mathrm{mm}^{2}$ (Landry, 2015). Since average lesion size is $\phi=20 \mathrm{~mm}^{2}$ (Stover, 1980), we let $b=10 \times 20 / 10=20$ per day and $s=200 \times 20 / 25=160$ per day. Oldest leaves may continue hosting sexual spore production even after they have fallen on the ground up to their disintegration (Stover, 1980 ), i.e. for several weeks. Hence $\mu$ may vary from $1 / 500 \approx 0.002$ to $1 /(12 \times 7) \approx 0.01$ per day. With such parameter values, $\mathscr{R}_{0}$ ranges from 1.5 to 2 . To conclude, considering that the number of neighbor sites $n$ ranges from 1 to $8, \sigma$ may vary from 0.8 to 6.5 per day.

\subsection{Bifurcation analysis}

All parameter values were fixed except $\mu$ and $\sigma$, the most uncertain parameters. A bifurcation analysis was conducted using XPP-AUTO (Ermentrout, 2002). Figure 5 shows that for parameter values realistic for the Black Sigatoka diseases of bananas, cyclic persistence occurs in a significant part of the parameter space.

\section{Discussion}

Epidemic thresholds The present study has shed light on the contribution of asexual and sexual spores in heterothallic fungal plant pathogens. It was shown specifically that with the production of both asexual and sexual spores from the same host, the basic reproduction number, $\mathscr{R}_{0}$, was unaffected by sexual reproduction. At very low densities the probability that two sexually compatible lesions interact is so low that sexual reproduction hardly occurs. Parasite dynamics thus essentially rely on asexual transmission and $\mathscr{R}_{0}$ is the same as in the SIR models. However, we also found that $\mathscr{R}_{0}$ cannot be confidently used as a threshold for epidemic development, since in species with low asexual $\left(\mathscr{R}_{0}<1\right)$ yet additional sexual reproduction capacities, noninfinitesimal initial parasite densities may generate epidemic outbreaks.

Cyclic dynamics Cyclic patterns of disease prevalence can emerge through different mechanisms such as (i) the interaction between demographic stochasticity and the inherent propensity of host-parasite dynamics to weakly dampened oscillations (Anderson et al, 1979), (ii) time-dependence of population parameters, (iii) intra-guild 


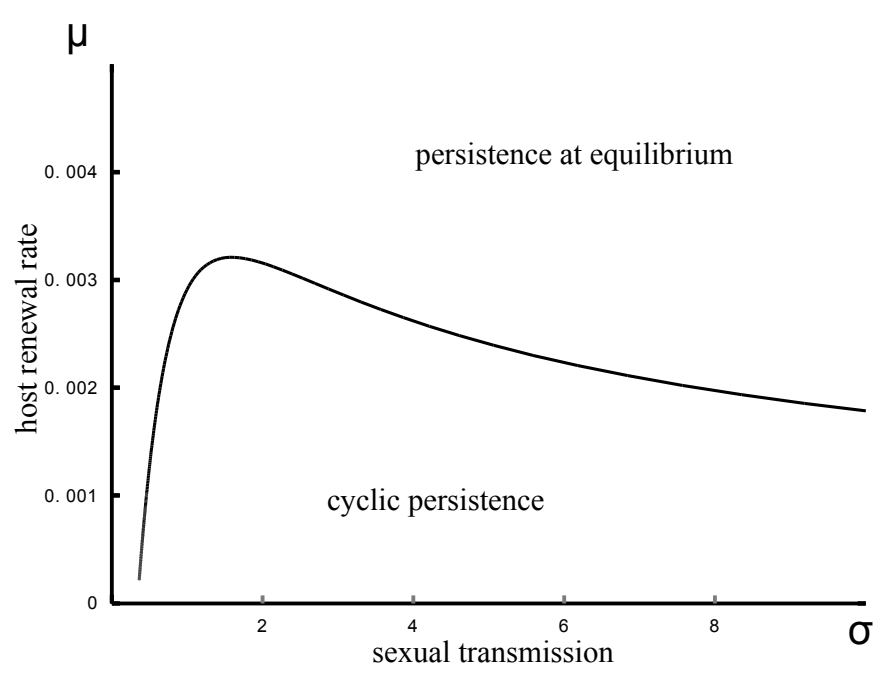

Fig. 5 Bifurcation analysis of the detailed model (10), as a function of sexual transmission $(\sigma)$ and host basic renewal rate $(\mu)$ with parameter values corresponding to Black Sigatoka (Section 3.2). Above the curve, the parasite can persist at equilibrium. Below the curve, there is cyclic persistence of the parasite.

predation among parasites infecting the same host (Hochberg and Holt, 1990), and (iv) "various kinds of nonlinearities in disease transmission terms" (Anderson et al, 1979). The present models do not incorporate (ii) or (iii) and clearly falls into the latter category of mechanisms, i.e., nonlinear transmission.

Without sexual spore production $(\sigma=0)$, the simple model has linear (asexual) transmission and never leads to limit cycles. Nonlinearities in transmission terms may result from a wide array of biological mechanisms, among which, non-homogeneous mixing of the host population, saturation of transmission rate when prevalence is high, free-living infective stages, or the impossibility of multiple infections (McCallum et al, 2001; Korobeinikov and Maini, 2005). It has been shown that in SIRS models with constant population size, the concavity of the transmission function relative to infected individuals density ensures the uniqueness and stability of the endemic equilibrium (Korobeinikov and Maini, 2005). In the present model, the transmission function $\left(\beta S I+\sigma S I^{2}\right)$ is convex in $I$, so that the endemic equilibrium might be unstable, leading to sustained oscillations (Zhang et al, 2016). Convex transmission arises when some form of cooperation is required for infection to occur, which is indeed what sexual reproduction implies.

Previous studies have focused on transmission terms of the form $S^{p} I^{q}$ (Liu et al, 1986; Hochberg, 1991). It was found that, for $p=1$ and $q=2$, i.e., strictly sexual species, cyclic dynamics were possible. Yet, we show here that cycles are non-generic in strictly sexual species, i.e., oscillations are confined to a thin range of parameters. Mixed reproduction enlarges the conditions for oscillations by allowing population growth at low densities. Thereby, even with high sexual transmission abilities, the parasite may alternate episodes of intense sexual spore production, which tend to 
over-exploit the host, with episodes where density has dropped and only asexual spore production is possible.

Many fungal plant diseases, including the Sigatoka diseases, and the South American leaf blight of rubber tree present sustained oscillations. Most of them are attributed to seasonal changes in climatic conditions (humidity, temperature, or both) or to temporal variability in the availability of susceptible host tissues. Management strategies consisting of roguing and replanting may also produce cyclic dynamics (van den Bosch and de Roos, 1996). In this study we provide a novel hypothesis to explain the cyclic nature of some epidemics for species capable of both sexual and asexual reproduction. However both previous studies and the present model suggest that correct epidemiological predictions will critically depend on the precise shape of the function that links infected density to transmission - called the mating function in two-sex models of population dynamics. In most heterothallic fungal pathogens, such data are currently missing.

Perspectives for Sigatoka diseases Using data from the literature, we parameterized the detailed model for the Black Sigatoka disease of bananas. From this very preliminary analysis, we suggest that $M$. fijiensis exhibits sufficient asexual reproduction to develop sustained epidemics starting from very low densities $\left(\mathscr{R}_{0} \approx 2\right)$. M. fijiensis has long been considered as relying mostly on sexual reproduction (Stover, 1980; Fouré, 1982). Accordingly, management practices include the removal of necrotic tissues, i.e. those that produce sexual spores. Yet our study suggests that hampering sexual reproduction is likely insufficient to eradicate the disease. Despite its deleterious consequences over yield, the removal of all wounded tissues (including leaves bearing lesions producing only asexual spores) should be considered as a complement to achieve eradication.

We also suggest that the species could well go through inherently sustained oscillations depending on environmental conditions and management practices. The Black Sigatoka does present cycles in disease severity. Whether these demographic variations correspond to inherently sustained oscillations caused by the mixed mating system or to variability in climatic conditions and management practices, is still unknown.

Moreover, until recently, it was generally considered that $M$. fijiensis excluded its sibling species, M. musicola, where both had been present, apart from elevated growing regions, where M. musicola persisted. However, these observations rely on very scarce data and it seems that in some places, $M$. fijiensis has been supplanted by $M$. musicola (Gomes et al, 2013). The present study suggests that competitive exclusion is not necessarily the rule, as two species can coexist on a single resource provided that one species oscillates autonomously (Armstrong and McGehee, 1980; Adler, 1990). Correctly predicting the year-to-year dynamics of the prevalence of both diseases would be of great help in optimizing the application of management practices. Nonetheless, achieving this goal will require obtaining long-term disease severity data on the scale of a growing region.

Conclusion Our study showed that precise knowledge of both sexual and asexual transmission abilities is required to predict the epidemiological dynamics of mixed 
mating species. Although M. fijiensis is one of the fungal heterothallic pathogens for which the most precise epidemiological data could be found, major gaps remained in our understanding of the pathogen biology. Spore infectivities, latency and infectious periods, lesion production rates need to be further documented. Beyond the present model, sexually and asexually produced spores often differ drastically in their epidemiological characteristics. For $M$. fijiensis for instance, sexually produced spores disperse from several hundred meters to several kilometers, whereas asexually produced spores travel only a few meters (Rieux et al, 2014). In other diseases it has been shown that the two mating types differed in their infectivity. From a theoretical perspective, this means that much work has to be done to understand the contributions of distinct types of spores to the epidemiological dynamics of fungal pathogens, integrating the key biological features of this important group of parasites.

Acknowledgements For this work, V.R. benefited from funds from project BIOFIS (reference 1001001) of the Agropolis Fondation (Montpellier, France). This work was supported by a grant overseen by the French National Research Agency (ANR) as part of the "Blanc 2013" program (ANR-13-BSV70011, FunFit project). F.H. also acknowledges partial funding from the Institut National de la Recherche Agronomique "Plant Health and the Environment" Division. We are grateful to C. Abadie, F. Bonnot, J. Carlier, C. Landry, S. Robert for biological discussions, to M. Baptiste for providing bibliographic material, to M. Castel, B. Facon, S. Gandon, O. Ronce, to Irma Mascio for help in the English editing, and to one anonymous reviewer for helpful comments.

\section{A Pair-formation, mating functions, and mate limitation}

In Section 2.3, we derived a bilinear (quadratic under even sex ratio) mating function from first principles focusing on plant pathogenic fungi. In this section, we discuss whether alternate mating functions could be considered to account for mate limitation in fungal plant parasites in general.

According to Hadeler (2012), a good mating function $\phi(x, y)$, where $x$ and $y$ are densities associated with both mating types, should satisfy the following conditions:

1. Preservation of positivity: $\phi(x, 0)=\phi(0, y)=0$ for all $x, y \geq 0$,

2. Homogeneity: $\phi(k x, k y)=k \phi(x, y)$ for $k \geq 0$,

3. Monotonicity: $u \geq 0, v \geq 0$ implies $\phi(x+u, y+v) \geq \phi(x, y)$.

Possible mating functions which satisfy these criteria include

- the geometric mean: $\phi(x, y)=(x y)^{1 / 2}$,

- the harmonic mean: $\phi(x, y)=2 x y /(x+y)$,

- the minimum: $\phi(x, y)=\min (x, y)$.

However, quoting Caswell (2001), "each of these function has been considered, and rejected by human demographers for one reason or another (Mc Farland, 1975), but the harmonic mean is regarded as the less flawed."

The bilinear mating function $\phi(x, y)=x y$ does not respect homogeneity since $\phi(k x, k y)=k^{2} x y$. Although this condition is indeed required for pair-formation, this does not invalidate our model since fungi and many other species with two mating types may not be monogamous, so that the mating function need not be restricted to pair formation.

Moreover, the homogeneity condition makes the function $\phi$ necessarily such that $\phi(x, x)=c x$ for some coefficient $c$ (Hadeler, 2012). In other words, such mating functions, per se, cannot account for mate limitation (or positive density dependence at low density), since the per capita mating rate is a constant $c$ under even sex-ratio. By contrast, the bilinear mating function yields a per capita mating rate with is increasing in population density: $\phi(x, x) / x=c x$ (Dennis, 1989).

In fact, we believe that the bilinear (or quadratic under even sex-ratio) mating function used in this study is the most natural one to model mate limitation, since it is simple and it can be derived from first principles; according to Dennis (1989), it was introduced by Volterra (1938). Note that Dennis (1989)'s 
caution that the quadratic (mate-limited) growth rate should not exceed the linear (mate unlimited) growth rate is easily taken into account by normalizing population density with respect to its carrying capacity, as naturally done in this study (i.e. in his notations, $\lambda \alpha n^{2} \leq \lambda n$ is ensured by taking $\alpha=1 / \bar{n}$, with $n \leq \bar{n}$ ). Also, the bilinear mating function is commonly used in the literature, e.g. (Veit and Lewis, 1996; Lehtonen and Kokko, 2011).

Actually, we simply used a cubic equation similar to the classical $\dot{n}=k n(n-a)(1-n)$, where $a$ represents an Allee effect threshold; this model is classically used to model mate-finding Allee effects in population dynamics (Lewis and Kareiva, 1993). Indeed, with $\alpha=0$, our strictly sexual model reads $\dot{I}=\sigma I^{2}(N-I)$ (since in this case $S=N-I$ ), which amounts to taking $a=0$ in the former model.

Extending our study to a negative exponential (Eq. 2) or to a rectangular hyperbola (Dennis, 1989) would be interesting but we do not think this would qualitatively change the results.

\section{References}

Adler F (1990) Coexistence of two types on a single resource in discrete time. Journal of Mathematical Biology 28(6):695-713

Anderson RM, May RM (1981) The population dynamics of microparasites and their invertebrate hosts. Philosophical Transactions of the Royal Society of London Series B, Biological Sciences pp 451-524

Anderson RM, May RM, et al (1979) Population biology of infectious diseases: Part I. Nature 280(5721):361-367

Arino J, McCluskey CC (2010) Effect of a sharp change of the incidence function on the dynamics of a simple disease. Journal of Biological Dynamics 4(5):490-505

Armstrong RA, McGehee R (1980) Competitive exclusion. American Naturalist pp 151-170

Billiard S, López-Villavicencio M, Devier B, Hood ME, Fairhead C, Giraud T (2011) Having sex, yes, but with whom? Inferences from fungi on the evolution of anisogamy and mating types. Biological Reviews 86(2):421-442

Bonner JT (1958) The relation of spore formation to recombination. American Naturalist pp 193-200

van den Bosch F, de Roos AM (1996) The dynamics of infectious diseases in orchards with roguing and replanting as control strategy. Journal of Mathematical Biology 35(2):129-157

Cannon P, Damm U, Johnston P, Weir B (2012) Colletotrichum current status and future directions. Studies in Mycology 73:181-213

Castel M, Mailleret L, Andrivon D, Ravigné V, Hamelin FM (2014) Allee effects and the evolution of polymorphism in cyclic parthenogens. American Naturalist 183(3):E75-E88

Caswell H (2001) Matrix population models. Wiley Online Library

Cornell SJ, Isham VS, Grenfell BT (2004) Stochastic and spatial dynamics of nematode parasites in farmed ruminants. Proceedings of the Royal Society of London, Series B: Biological Sciences 271(1545):1243-1250

Dennis B (1989) Allee effects: population growth, critical density, and the chance of extinction. Natural Resource Modeling 3(4):481-538

Dhooge A, Govaerts W, Kuznetsov YA, Meijer H, Sautois B (2008) New features of the software MatCont for bifurcation analysis of dynamical systems. Mathematical and Computer Modelling of Dynamical Systems 14(2):147-175

van den Driessche P, Watmough J (2008) Further notes on the basic reproduction number. In: Mathematical Epidemiology, Springer, pp 159-178

Ene IV, Bennett RJ (2014) The cryptic sexual strategies of human fungal pathogens. Nat Rev Microbiol 12:239-251

Ermentrout B (2002) Simulating, analyzing, and animating dynamical systems: a guide to XPPAUT for researchers and students, vol 14 . SIAM

Fouré E (1982) Les cercosporioses du bananier et leurs traitements. comportement des variétés. 1: Incubation et évolution de la maladie. Fruits 37(12):749-766

Gomes LIS, Douhan GW, Bibiano LB, Maffia LA, Mizubuti ES (2013) Mycosphaerella musicola identified as the only pathogen of the sigatoka disease complex present in Minas Gerais State, Brazil. Plant Disease 97(12):1537-1543

Gourbière S, Gourbière F (2002) Competition between unit-restricted fungi: a metapopulation model. 217:351-368

Hadeler K (2012) Pair formation. Journal of Mathematical Biology 64(4):613-645 
Hamelin FM, Castella F, Doli V, Marçais B, Ravigné V, Lewis MA (2016) Mate finding, sexual spore production, and the spread of fungal plant parasites. Bulletin of Mathematical Biology 78(4):695-712

Hochberg ME (1991) Non-linear transmission rates and the dynamics of infectious disease. Journal of Theoretical Biology 153(3):301-321

Hochberg ME, Holt RD (1990) The coexistence of competing parasites. I. The role of cross-species infection. American Naturalist pp 517-541

Jordan CF (1969) Derivation of leaf-area index from quality of light on the forest floor. Ecology pp 663 666

Korobeinikov A, Maini PK (2005) Non-linear incidence and stability of infectious disease models. Mathematical Medicine and Biology 22(2):113-128

Kuznetsov IA (1998) Elements of applied bifurcation theory, vol 112. Springer

Landry C (2015) Modélisation des dynamiques de maladies foliaires de cultures pérennes tropicales différentes échelles spatiales : cas de la cercosporiose noire du bananier. $\mathrm{PhD}$ thesis, Université des Antilles

Lehtonen J, Kokko H (2011) Two roads to two sexes: unifying gamete competition and gamete limitation in a single model of anisogamy evolution. Behavioral Ecology and Sociobiology 65(3):445-459

Lewis M, Kareiva P (1993) Allee dynamics and the spread of invading organisms. Theoretical Population Biology 43(2):141-158

Liu Wm, Levin SA, Iwasa Y (1986) Influence of nonlinear incidence rates upon the behavior of SIRS epidemiological models. Journal of Mathematical Biology 23(2):187-204

May R, Woolhouse M (1993) Biased sex ratios and parasite mating probabilities. Parasitology 107(03):287-295

May RM (1977) Togetherness among schistosomes: its effects on the dynamics of the infection. Mathematical Biosciences 35(3):301-343

Mc Farland DD (1975) Models of marriage formation and fertility. Social Forces 54(1):66-83

McCallum H, Barlow N, Hone J (2001) How should pathogen transmission be modelled? Trends in Ecology \& Evolution 16(6):295-300

Nåsell I (1978) Mating models for schistosomes. Journal of Mathematical Biology 6(1):21-35

Nåsell I, et al (1985) Hybrid models of tropical infections. Lecture Notes in Biomathematics (59)

Nirenberg H, O'Donnell K (1998) New Fusarium species and combinations within the Gibberella fujikuroi species complex. Mycologia 90(3):434-458

Reluga TC, Medlock J, Perelson AS (2008) Backward bifurcations and multiple equilibria in epidemic models with structured immunity. Journal of Theoretical Biology 252(1):155-165

Rieux A, Soubeyrand S, Bonnot F, Klein EK, Ngando JE, Mehl A, Ravigné V, Carlier J, de Lapeyre de Bellaire L (2014) Long-distance wind-dispersal of spores in a fungal plant pathogen: estimation of anisotropic dispersal kernels from an extensive field experiment. PloS ONE 9(8):e103,225

Robert S (2012) Emergence mondiale de la maladie des raies noires du bananier: histoire de l'invasion et stratégie de vie du champignon phytopathogène Mycosphaerella fijiensis. $\mathrm{PhD}$ thesis, Université de Montpellier 2

Robert S, Ravigné V, Zapater MF, Abadie C, Carlier J (2012) Contrasting introduction scenarios among continents in the worldwide invasion of the banana fungal pathogen Mycosphaerella fijiensis. Molecular Ecology 21(5):1098-1114

Rodríguez-Guerra R, Ramírez-Rueda M, M CE, García-Serrano M, Lira-Maldonado Z, Guevara-González R, González-Chavira M, Simpson J (2005) Heterothallic mating observed between mexican isolates of Glomerella lindemuthiana. Mycologia 97:793-803

Savary S, Stetkiewicz S, Brun F, Willocquet L (2015) Modelling and mapping potential epidemics of wheat diseases-examples on leaf rust and Septoria tritici blotch using EPIWHEAT. European Journal of Plant Pathology 142(4):771-790

Steenkamp E, Wingfield B, Coutinho T, Zeller K, Wingfield M, Marasas W, Leslie J (2000) PCR-based identification of MAT-1 and MAT-2 in the Gibberella fujikuroi species complex. Applied and Environmental Microbiology 66(10):4378-4382

Stover R (1980) Sigatoka leaf spots of bananas. Plant Disease 64(8):751

Turner DW, Fortescue JA, Thomas DS (2007) Environmental physiology of the bananas (Musa spp.). Brazilian Journal of Plant Physiology 19(4):463-484

Veit RR, Lewis MA (1996) Dispersal, population growth, and the Allee effect: dynamics of the house finch invasion of eastern north america. American Naturalist pp 255-274

Volterra V (1938) Population growth, equilibria, and extinction under specified breeding conditions: a development and extension of the theory of the logistic curve. Human Biology 10(1):1-11 
Watling R, Frankland J, Ainsworth A, Isaac S, Robinson C (2002) Tropical mycology

Williams GC (1975) Sex and evolution. 8, Princeton University Press

Zadoks J (1971) Systems analysis and the dynamics of epidemics. Phytopathology 61:600-610

Zhang W, Wahl LM, Yu P (2016) Backward bifurcations, turning points and rich dynamics in simple disease models. Journal of Mathematical Biology pp 1-30 